\title{
Clinical Feature and Initial Treatment Strategy of Laryngeal Obstruction in the Neck
}

\author{
Shujun Sun ${ }^{1}$, Limin Zhao ${ }^{1}$ \\ ${ }^{1}$ Affiliated Hospital of Weifang Medical University,Weifang,261030,China
}

Keywords: Laryngeal obstruction; neck trauma; tracheotomy

\begin{abstract}
Objective To explore the clinical feature and initial treatment strategyof laryngeal obstruction in the neck trauma. And analysis clinical incidence rate and etiological factors for effective remedy and prevention and cure of the disease. Method A total number of 85 cases were seen All were patients whose age range was from 6 years to 72 years with a mean age of 28.2 years.closed 52 cases and opened neck trauma 33cases; combined type 15 cases;road accident 33 cases; lance trauma 18 cases etc. Results About $1.7 \%$ of the total patients were neck trauma. within the patients about $61.2 \%$ was closed neck trauma;38.8\% opened neck trauma;17.6\% combined type trauma ;38.8\% road accident; complicated laryngeal obstruction in the closed neck trauma was 44 cases; 14 cases in opened neck trauma.the clinical expression of laryngeal obstrucion divided into the acute and lately stage.Treachotomy plays important roles in the treatment of laryngeal obstrucion.but in the lately stage appear the complications of deadth and abscess of neck hemorrhagic shock . Patients with attempted suicide had deep open wounds exposing the pharynx, larynx or both with horizontally positioned incision wounds while homicidal cases had obliquely positioned incision wounds. Restoration of normal neck, pharyngeal and laryngeal architecture was carried out through meticulous surgical repair of various tissue layers with nasogastric tube in situ for 7-9 days. Conclusion Significant proportion of emergencies from anterior neck cut throat injuries in this study. Prompt surgical repair and concurrent psychiatric evaluation in attempted suicide and homicide is required. Treachotomy plays important roles in the treatment of laryngeal obstrucion in the early stage.Doctor takes attention of the laryngeal obstruction which appears in the lately stage.The etiology of death and complications lie in the not obvious clinical expresions and symptoms which cover up the situations of patients and produce diagnostic errors.
\end{abstract}

\section{Introduction}

Common clinical neck trauma is a serious illness due to the rich vascular neck and airway and cause, neck trauma can be divided into open and closed two categories, which closed is more caused by boxing, sticks and car crash and other impact. Compared with open wounds, sometimes closed wounds cannot be ignored. This is because of blunt trauma after the injury without signs of obvious symptoms. Many patients can lead to acute laryngeal obstruction, hemorrhagic shock, late scarring and other severe tracheal stenosis complications. Our department collected 85 cases of hospitalized patients with neck trauma since January 2005. Neck trauma through 85 cases of patients with sorting, statistics, analysis otolaryngology head and neck trauma and laryngeal obstruction disease clinical features and treatment strategies, and analysis of such diseases clinical morbidity and risk factors for the effective treatment and prevention help.

\section{Clinical data}

1.1 General information: General information: male 71 cases, 14 females, aged 6 years old -72 years old, 6-12 years old 3 cases; 13-18 years 14 cases; 19-30 years old 27 cases; 31-55 years old 23 cases; 56 -72 years old the age of 18 cases

1.2 Pathogenic factors: 52 cases of blunt trauma patients, open 33 cases, 15 cases in which the combined injury, traffic accident in 33 cases, 18 cases of knife wounds, Boynton wounded (boxing or sticks injured) 34 cases. 
1.3 Clinical features: Closed injury early in 52 cases of hoarseness, aphasia, different degree of difficulty in breathing, not eating, coughing up blood and sputum, neck tenderness obviously, with varying degrees of neck subcutaneous emphysema. Based on patients with early stage laryngeal obstruction appearance, which can be divided into two categories: one early without difficulty breathing, difficulty in breathing the next day or every other day, 8 cases of early hoarseness, throat obstruction in 44 cases, 13 cases with not eating, with hemoptysis and sputum 49 cases; the other has a different degree neck tenderness, subcutaneous emphysema in severe cough, cough after injury in a car accident injuries and sticks are more common. After examination revealed the patient who can not eat is with esophageal inlet or aryepiglottic fold, arytenoid injury; patient developed severe coughing and coughing up sputum, throat and mucous membrane bleeding due to breathing difficulties and tear occurs, oozing secretions can not swallow cause choking. Subcutaneous emphysema patients with severe impacts throat and neck cause severe mucosal tear gas into the subcutaneous severe coughing, subcutaneous emphysema, 28 cases occurred in 52 cases of blunt neck trauma, accounting for $53.8 \%$.

Late onset of laryngeal obstruction in patients with early is diagnosis by voice hoarse, no difficulty breathing or mild dyspnea, the patients with laryngeal cavity bleeding after mucosal edema, severe difficulty breathing, 3 cases died, 2cases in 3 with multiple injuries combined craniocerebral trauma or head facial trauma, 1 case of patients with compound wound analysis of death cause delay of laryngeal obstruction that doctors have no attention, acute onset of laryngeal cavity ooze blood or blood vessels rupture with laryngeal cavity mucosal edema cause irreversible damage. Early acute laryngeal obstruction in clinical sometimes is not fearful, then the trachea incision and laryngeal cavity ploration life to win the precious time to rescue patients.

Open neck trauma is common in commit suicide, traffic accident and the knife cut, including 11 cases with head facial trauma, knife cut with neck vascular rupture in 5 cases of hemorrhagic shock, 2 cases for rescue invalid death. There are 8 patients combined injury because of unconsciousness with head injury. There are 22 cases of patients without combined wound with different degree of blood, hoarse and has lost his voice, laryngeal obstruction, and the clinical manifestations such as subcutaneous emphysema. Knife cuts including suicide in patients with artery on the neck caused by knife cut throat or most common thyroid artery rupture, and the external carotid artery rupture is the most rare.开放 Hoarseness accounted for 16 cases (16/33, 48.5\%), and blunt trauma in 46 cases (46/52, 88.5\%), the difference was significant between openness and neck trauma. Analyze its principle is mainly caused by blunt trauma. Boynton wounded may cause vocal cord edema and loss of different degrees of hoarseness. The openness of trauma due to a sharp tool to cut the neck nerves before a hoarse voice, and the recurrent laryngeal nerve injury is aspect cannot be ignored.

\section{Initial treatment strategie}

We are following an important principle in the rescue of patients with neck trauma which is found early laryngeal obstruction. Early tracheotomy has brought great convenience for patients after illness recovery, for the patients with improved breathing difficulties, and subcutaneous emphysema, clearing secretions, played an important role in hemostasis. Some physicians for patients with nocturnal visits, failed to timely tracheotomy patients delay. 52 cases of closed injury of laryngeal obstruction 44 cases, 28 patients with tracheotomy, 1 cases with late appearance of laryngeal obstruction is not in a timely manner tracheostomy laryngeal cavity bleeding more deaths. Blunt trauma due to the merger throat and neck edema edema, oozing mainly large doses of hormones and antibiotics, oxygen inhalation is crucial to alleviate the condition. There are 16 cases without laryngeal obstruction tracheotomy patients were treated conservatively, with hormones, antibiotics therapy, adjuvant hormonal spray, oxygen inhalation, intravenous rehydration therapy. There are 13 patients who cannot eat or swallow saliva should cause enough attention, ring dipper joint damage, scoop epiglottis plica damage or fracture edema in patients with esophageal entrance injury, or even can not eat, the early insert gastric tube is conducive to improve the condition of patients can not eat late. We treat the patients who can't eat, patients with swallowing choking 
cough cannot cooperate with stomach tube inserted, stomach tube insertion under general anesthesia in time to overcome the wound after not eating simply rely on intravenous fluids to maintain fluid balance. Cough, sputum is the most common clinical manifestations of cough medicine only as adjuvant therapy, early treatment can not cough medicine, it will mask the disease, delaying treatment.

Open neck injury to the upper neck wound common, timely bleeding, hemorrhagic shock is crucial rescue. Exploration and throat tracheotomy can be performed simultaneously, help stop the bleeding edge side of the tracheal tube insertion. But clinically sometimes it is difficult to do, the first consideration is to stop the bleeding while rehydration request anesthesiologist intubated, connected to the lower end of a one-time blood balloon catheter prevention throat or neck into the trachea. There are 33 cases of open neck trauma patients in a timely manner the neck vascular ligation hemostasis, 21 patients of laryngeal artery rupture, the rest for the fracture of external carotid artery, 5 cases with damage heavier uncontrolled hemorrhagic shock, 2 cases with craniocerebral trauma and facial multiple losses and more laryngeal cavity bleeding caused by acute laryngeal obstruction leads to death. Find bleeding blood vessel rupture hemostasis is the key to treatment, because the majority of patients with laryngeal obstruction, tracheal tube insertion time for effective hemostasis and rehydration can reduce the number of death cases. External carotid artery rupture or loss often merge on laryngeal nerve damage, patients with laryngeal cavity mucosa hypoesthesia. Large area of laryngeal cavity merging neck para thyroid injury bleeding and layer suture prevention and treatment of postoperative laryngeal cavity and legacy is an aspect of attention. We use of 7 patients with large area of laryngeal cavity damage expansion 3-6 months, 1 cases colostomy, the trachea cut due to eliminate the thyroid cartilage, laryngeal cavity severe stenosis, can't tube drawing.

\section{Conclusion}

Otolaryngology head and neck trauma is common, because of the complexity and importance of the neck anatomical characteristics, treatment issues its combined injury, is particularly important. The clinical features of the high incidence of the disease complex after injury, clinical manifestation, treatment to bring greater difficulty, not only to neck trauma, but also to associated with head and facial vascular injury, hemorrhagic shock, coma, laryngeal obstruction, facial bone fracture, nerve damage, hearing vestibular dysfunction, language swallowing function. So in the early treatment should be based on head and neck anatomy and injury characteristics, must conduct a comprehensive inspection and analysis, arrange emergency treatment priorities. For life-threatening wounds, should be timely emergency treatment, including keeping the airway open and timely prevention of shock, bleeding, through consultation between subjects, do not misdiagnosis and missed diagnosis. In salvage therapy at the same time, should also pay attention to the function of head and neck injuries, especially laryngeal airway function after healing effect, prevent to produce the serious complications such as laryngeal airway stenosis. Division by 85 cases my department deal with has different levels of treatment and neck trauma associated with laryngeal obstruction in patients, found in favor of early tracheotomy to restore the patient's condition, to avoid serious complications such as subcutaneous emphysema. Severe oral mucosa damage the thyroid cartilage tear and also pay attention to try to keep the thyroid cartilage of the larynx to prevent postoperative stenosis, dilation son pulled caution. The domestic many authors agree to the above point of view, Kong Yonggang[1].etc think the laryngeal wound closure must pay attention to the function of larynx, soft reset, damaged mucous membrane suture and laryngeal mucous membrane, cartilage and damaged trachea cartilage should try to retain, strict registration suture, severe fracture, throat cavity lien mode after reset, cartilage surface overlapping bartackings muscles. The annular scoop we joint dislocation treatment in patients with early in order to improve breathing difficulties, for intubation in patients with severe treatment, laryngeal obstruction after tracheotomy. But not in patients with acute laryngeal obstruction to manipulate the cricothyroid membrane puncture. Patients found to be badly in need of improvement of oxygen environment, such as Wangwei[2] 
first adopted the cricothyroid membrane puncture outfit line cricothyroid membrane puncture, quick, safe, reliable results have been achieved, the patients of laryngeal cavity hemorrhage complicating acute laryngeal obstruction to the anesthesiologist cooperate with cricothyroid membrane puncture is also a kind of good method. Neck trauma combined laryngeal obstruction patient most can't eat, it is difficult to keep effective fluid balance by intravenous drip rehydration, 1 cases of laryngeal obstruction and scoop epiglottis plica, esophageal entrance injury patients in general anesthesia downlink stomach tube implantation, through BiSi patients recover quickly. Tracheal tube should carefully pull out, not simply pull out according to time to determine whether the tracheal tube, especially for patients with laryngeal and tracheal angioplasty better to be cautious.

O.L angeron, etc. [3] made many researches to keep airway unobstructed and pointed out the important role of neck trauma to keep airway unobstructed difficulty as the vital signs of instability and increased the degree of difficulty in breathing, but combination of cervical spine injury even if early endotracheal intubation may not be successful. Zhang Xiaofan [4] believes that the use of noninvasive positive pressure ventilation pre-hospital long-distance transfer and shortness of breath and severe neck trauma patients is feasible, but strict inclusion criteria, fully prepared before forwarding, close observation is the key to the safe transfer. Cervical trauma merge laryngeal obstruction in patients with airway processing need a variety of effective cooperation: good laryngoscope visible light source, light operation skill and familiar with the oropharynx, anatomical structure, etc., such as merger serious loss not impose intubation of cervical vertebra, can also be prepared for the cricothyroid membrane puncture, this requires multidisciplinary work closely and fully consider the severity of the patient condition.

\section{References}

[1] Kong Yonggang, Yu Rong prostitutes, Liugong Han. Neck opening 28 cases of sexual trauma treatment [J]. Chinese Journal of Otorhinolaryngology skull base, 1999,5 (3) :153-155.

[2] Wang Wei Li, Qiao Yan Wen Clinical observation Thyrocricocentesis is rescued laryngeal obstruction [J] Chinese Critical Care Medicine, 1997,17 (6): 50.

[3] O.Langeron,A.Biernabum,J.Amour.Airway management in trauma[J]. MINERVA ANESTESIOL,2009,75:307-11.

[4] Zhang Fan, The application in front of noninvasive positive pressure ventilation in critically ill patients with dyspnea long hospital transfer [J] Chinese Critical Care Medicine, 2008,28 (11): 1042-1044. 